\title{
How we achieved $0 \%$ of thrombotic graft loss: the initial 60 cases of pancreas transplant
}

\author{
Byung Hyun Choi
}

Department of Surgery, Pusan National University Yangsan Hospital, Yangsan, Korea

Background: One of the major surgical complications after pancreas transplant is early graft failure due to thrombosis. Unfortunately, the early graft loss rate has not improved over the last 30 years. Even in recent reports, the technical failure rate due to thrombosis was $5 \%-10 \%$. The low flow portal venous system must have the tendency of thrombosis, thus the graft pancreas in inherently thrombogenic. Therefore, wider and shorter anastomosis of the portal vein is better for maintaining blood flow. The size of the opening is more important than the length according to the equation above.

Methods: We performed 60 cases of pancreas transplant (11 simultaneous pancreas-kidney, or simultaneous pancreas living-donor kidney, 14 pancreas after kidney, 34 pancreas transplant alone, and one pancreas after liver transplant) in Pusan National University Yangsan Hospital, Korea, since 2015. There is no thrombotic graft loss in our cases.

Results: Most of our cases were performed with venous drainage to vena cava and duodenal exocrine drainage. The venous anastomotic technique was evolved from the direct anastomosis $(n=8)$. Then we did a diamond-shaped patch anastomosis $(n=13)$. After that, the fence angioplasty with using vena cava graft was applied to 31 cases. And finally, we have been performing the venous anastomosis with aortic interpositional graft $(n=8)$. The conventional or low molecular heparin was used for the prevention of thrombosis in the first 40 cases of transplant. However, we do not use any heparin anymore neither in the intraoperative nor postoperative periods. There was some partial thrombosis in graft splenic vein or superior mesenteric vein on computed tomography scans. However, these partial thromboses did have any effect on graft loss.

Conclusions: In conclusion, we need to understand the mechanism of thrombus in graft pancreas and should do the best effort to make the wide venous anastomosis and maintain the venous flow. We would like to share our know-how with members of transplant society.

Corresponding author: Byung Hyun Choi

E-mail: gmoolpop@gmail.com

(c) The Korean Society for Transplantation

This is an Open Access article distributed under the terms of the Creative Commons Attribution Non-Commercial License (http://creativecommons.org/licenses/by-nc/4.0/) which permits unrestricted non-commercial use, distribution, and reproduction in any medium, provided the original work is properly cited. 